\title{
Wenn Bürger nicht nur Wähler sein wollen. Elektronische Demokratie in Südkorea - ein Testfall*
}

\author{
Eun-Jeung Lee
}

Südkorea bietet einen interessanten Testfall für die Analyse von elektronischer Demokratie („e-democracy“). Es verfügt über eine sehr gut ausgebaute technische Internetinfrastruktur ${ }^{1}$ und zeichnet sich durch eine äußerst aktive Nutzung des Internets durch Bürger und Politiker $^{2}$ aus. ${ }^{3}$ Besonders bemerkenswert sind online-basierte Formen der politischen Netzwerkbildung. Die Bürger, die allgemein „Netizen“ (Net-Citizen) genannt werden, mobilisieren sich selbst spontan-,, chaotisch“ und werden zum kollektiven Kontrolleur von Politik, Politikern und Staat. Innerhalb der interaktiven Kommunikationsstruktur des Web 2.0 bestimmen sie selbst die Themen und organisieren ihre eigenen offline Veranstaltungen. ${ }^{4}$ Der Prozess ist nicht steuerbar und kann durch gezielte Platzierung von Informationen auf Websites kaum beeinflusst, geschweige denn gesteuert werden. Die Online- und OfflineAktivitäten wirken aufeinander, und ihre Grenzen sind fließend. Die über viele Wochen andauernden Kerzendemonstrationen im Frühjahr 2008, zu denen landesweit an manchen Tagen über eine Million Menschen zusammenkamen, sind durch die interaktiven Strukturen des Web 2.0 und die Verzahnung der Online- und Offline-Aktivitäten zu erklären.

\section{Theoretische Überlegungen}

Die dynamische Entwicklung des Internets hat die Welt der Politik und der Bürger beträchtlich verändert. ${ }^{5}$ In den letzen Jahren sind zahlreiche Studien, Bücher und Aufsätze

* Die vorliegende Arbeit wurde durch die Academy of Korean Studies der Republik Korea im Jahr 2009 unterstützt (AKS-2009-MA-1001).

1 Der Verbreitungsgrad des Internets hat sich in Korea in den 2000er Jahren stetig erhöht. 2008 gab es 35.360.000 Internetnutzer; die Internetnutzungsrate beträgt 77,1 Prozent; vgl. National Internet Development Agency of Korea, NIDA 2008, S. 21.

2 Bei der Präsidentschaftswahl 2004 verfügten von den 1.175 Kandidaten bereits 85,3 Prozent über eine eigene Homepage im Internet; vgl. Tong-Jin Pak, ch'ongsŏn-gwa int'ŏnet [Parlamentswahl am 15. April 2004 und Internet], Vortragsmanuskript, Tagung „Internet und Parlamentswahl am 15. April“, Bürgerorganisation Goodcitizen, Seoul 2004, S. 6, http://www.goodcitizen.or.kr/pds/ upload/ (Abruf am 10. Januar 2005).

3 Statistiken der National Internet Development Agency of Korea zeigen, dass 2008 die durchschnittliche Nutzungszeit des Internets pro Woche 13,7 Stunden betrug; vgl. National Internet Development Agency of Korea, NIDA 2008, S. 30.

4 Vgl. Bruce Bimber, Information and American Democracy. Technology in the Evolution of Political Power, Cambridge 2003.

5 So schrieb Bruce Bimber mit Bezug auf die USA: „Over the space of about five years, we have witnessed the adoption of new means for communication and management of information by virtually every political organization and institution of consequence in the country $[\ldots]$ at no time in the history of American democracy has as new set of communication and informationhandling capacities been assimilated so rapidly by the political system.", Bruce Bimber, a.a.O. (Fn. 4), S. 1. 
über die Auswirkungen des Internets auf Politik und politische Prozesse erschienen. ${ }^{6}$ Dennoch wird das Internet selbst unter Politikwissenschaftlern immer noch als ein weitgehend technisches Medium für billige, einseitige Informationsverbreitung betrachtet. ${ }^{7}$ Seine Besonderheit liegt aber in der Reziprozität der Massenkommunikation - und dies gilt umso mehr, als sich in den letzten Jahren das Web 2.0 verbreitet hat. Dadurch ist eine neue Art von Öffentlichkeit, nämlich eine „eletronic agora“, ein elektronischer Marktplatz, entstanden. ${ }^{8}$

Möglicherweise ist diese Besonderheit, nachdem die anfängliche Euphorie über die Auswirkungen des Internet auf Politik und politische Prozesse Skepsis und Kritik gewichen ist, wieder in Vergessenheit geraten. Von den Hoffnungen, mit Hilfe des Internets eine starke Demokratie zu verwirklichen ${ }^{9}$, politische Ungleichheit durch besseren Zugang zu Informationen zu überwinden ${ }^{10}$ oder die Gemeinschaft und den Gemeinschaftsgeist zu stärken ${ }^{11}$, ist nicht mehr viel zu hören. Stattdessen überwiegt in der akademischen Debatte die Sicht der Skeptiker, dass nämlich eine neue Technologie allein nicht bewirken könne, dass Menschen, denen es an Interesse für Politik, Gesellschaft und Staat mangelt, sich plötzlich dafür interessieren oder gar sich engagieren. ${ }^{12}$ Zudem habe sich gezeigt, dass mit der fortschreitenden Kommerzialisierung des Internets Strukturen herausgebildet wurden, über die Informationen selektiert, kanalisiert und auch manipuliert werden. Diese Entwicklungen fanden ihren Niederschlag im Begriff der „digital divide“, deren Kernaussage darin besteht, dass in der Nutzung des Internets entlang soziodemographischer Merkmalsausprägungen wie Geschlecht, Einkommen oder Schulbildung erhebliche Unterschiede festzustellen sind. ${ }^{13}$

6 Einen guten Überblick dazu gibt Andrew Chadwick, Internet Politics: States, Citizens, and New Communication Technologies, New York 2006; vgl. auch Keith N. Hampton, Grieving for a Lost Network: Collective Action in a Wired Subburth, in: The Information Society, 19. Jg. (2003), H. 5, S. 417 - 428; Kevin A. Hill / John E. Hughes, Cyberpolitics: Citizen Activism in the Age of Internet, Rowman / Littlefield 1998; Bruce Bimber, a.a.O. (Fn. 4); Pippa Norris, The Digital Divide: Civic Engagement, Information Poverty, and the Internet Worldwide, New York 2001; Richard Davis, The Web of Politics: The Internet's Impact on the American Political System, New York 1999; Barry Wellman / Caroline A. Haythornthwaite, The Internet in Everyday Life, Malden 2002.

7 Ein Beispiel dafür bietet ein Artikel in der Frankfurter Allgemeine Zeitung. Unter dem Titel „Präsident 2.0" heißt es dort: Barack Obama besitze einen eigenen Blog, seine Unterstützer fänden sich auf seiner Homepage zusammen; er wolle auch nach der Wahl vom Weißen Haus aus mit den Bürgern kommunizieren. „Obama (könnte) mit Hilfe seiner Datenbank ohne weiteres Millionen Unterstützer mobilisieren." Stefan Tomik, Präsident 2.0, in: FAZ vom 20. Januar 2008, S. 3 .

8 Vgl. Howard Rheingold, The Virtual Community. Homesteading on the Electronic Frontier, Reading / Addison-Wesley 1993.

9 Vgl. Benjamin R. Barber, Strong Democracy, Berkeley 1984.

10 Vgl. Robert Dahl, Democracy and Its Critics, New Heaven 1989.

11 Vgl. Amitai Etzioni, The Spirit of Community: Rights, Responsibilities, and the Communitarian Agenda, New York 1993.

12 Vgl. Richard Davis, a.a.O. (Fn. 6); Michael Margolis / David Resnick, Politics as Usual: The Cyberspace Revolution, London 2000; Pippa Norris, A Virtuous Circle: Political Communications in Postindustrial Societies, Cambridge 2000.

13 Vgl. Mark Warschauer, Technology and Social Inclusion: Rethinking the Digital Divide, Cambridge 2003; Karen Mossberger / Caroline J. Tolbert / Mary Stansbury, Virtual Inequality: Beyond the Digital Divide, Washington D.C. 2003; Eszter Hargittai / Gina Walejko, The Participation 
Es ist interessant zu beobachten, wie stark sich die Skepsis gegenüber dem Internet als eine neue Kommunikationstechnologie verbreitet hat. Nicht nur im Kontext der akademischen Diskurse über die digital divide, sondern auch in der öffentlichen Debatte, insbesondere unter Intellektuellen in Europa, bleibt die Einstellung gegenüber dem Internet überwiegend kritisch, bis hin zu der Behauptung, dass es schlichtweg nichts mit Demokratie zu tun habe und von elektronischer Demokratie erst gar nicht die Rede sein könne. In der Debatte um das Verhältnis von Internet und Politik, die im Mai und Juni 2009 in der Wochenzeitung Die Zeit geführt wurde, argumentiert Jens Jessen sogar, dass das Internet lediglich ein Hort des Populismus sei. Er kritisiert diejenigen, die darin ein egalitäres Kommunikationsmedium zur Bildung der öffentlichen Meinung sehen, als Netfanatiker. ${ }^{14}$

Es ist nicht zu leugnen, dass die Verbreitung des Internets auch populistische Risiken und die Verbreitung von Gerüchten mit sich bringt. Solche Negativaspekte sind jedoch nicht nur bei den neuen Kommunikationstechnologien, sondern auch bei allen anderen Medien festzustellen. Auch Zeitung, Rundfunk und Fernsehen können auf Politik und Demokratie positive wie negative Einflüsse ausüben. Warum also sollten nur die problematischen Wirkungen des Internets hervorgehoben werden?

In Bezug auf die Entwicklung neuer Kommunikationstechnologien war schon immer zu beobachten, dass zunächst mit mehr oder weniger großer Skepsis reagiert wurde, bevor man überhaupt damit anfing, sich über die politischen Potentiale der neuen Technologie ernsthaft Gedanken zu machen. Wie Bruce Bimber gezeigt hat, hat die Entwicklung der Kommunikationstechnologien letztlich zur Entfaltung der Demokratie Wesentliches beigetragen. Das Internet bildet in dieser Hinsicht keine Ausnahme. ${ }^{15}$

Möglicherweise ist es diese ganz neue Art der Kommunikation und Informationsverbreitung, die zugleich das Monopol der geistigen Eliten, der „Geistesaristokratie“16, in Frage stellt und die Skepsis der Intellektuellen umso stärker beflügelt. Das kann aber kein Grund sein, sich in der Debatte um neue Kommunikationstechnologien und Politik beziehungsweise Demokratie lediglich auf deren negative Seiten zu konzentrieren. So können auch positive Auswirkungen des Internets auf die Demokratie am Beispiel der südkoreanischen Erfahrungen seit 2000 gezeigt werden.

Die Existenz der digital divide scheint durch die von Barabasi und Albert formulierte These der „power law distribution“ im World Wide Web untermauert zu werden: „Because of the preferential attachment, a vertex that acquires more connections than another one will increase its connectivity at a higher rate; thus, an initial difference in the connectivity between two vertices will increase further as the network grows. [...] Thus older [...] vertices increase their connectivity at the expense of the younger [...] ones, leading over time to some vertices that are highly connected, a ,rich-get-richer' phenomenon. " ${ }^{17}$ Das Phänomen, dass „die Reichen reicher werden“, wird von diesen Autoren vor allem durch die bes-

Divide: Content Creation and Sharing in the Digital Age, in: Information, Communication and Society, 11. Jg. (2008), H. 2, S. 239 - 256; Pippa Norris, a.a.O. (Fn. 6).

14 Vgl. Jens Jessen, Das Netz trügt, in: Die Zeit vom 4. Juni 2009, http://www.zeit.de/2009/24/Internetdebatte (Abruf am 28. Juni 2009).

15 Vgl. Bruce Bimber, a.a.O. (Fn. 4).

16 Kang Wón T’aek, Internet-gwa han'guk chŏngch'i [Internet und Politik in Korea], Seoul (nanam) 2008, S. 59.

17 Albert-Laszlo Barabasi / Reka Albert, Emergence of Scaling in Random Networks, in: Science, 286. Jg. (1999), H. 5439, S. $509-512$. 
sere Sichtbarkeit, die unter anderem ein Ergebnis der attraktiven Gestaltung von Websites und der Investition in Zeit und Geld ist, begründet. Wie die südkoreanischen Erfahrungen der letzten Jahre allerdings zeigen, sind es gar nicht so sehr die Sichtbarkeit und die Gestaltung von Informationen, sondern vielmehr die Inhalte, die für das Ausmaß der Informationsdiffusion im Internet entscheidend sind. Wie noch am Beispiel der Online-Petition für die Amtsenthebung von Präsident Lee Myung-Bak im April 2008 zu zeigen sein wird, kann in der Online-Öffentlichkeit selbst eine ganz einfach gestaltete Website oder ein ganz schlichter Eintrag eines einzelnen Bürgers große Resonanz finden, also ebenfalls ein „Reich wird reicher"-Phänomen hervorbringen.

Dies ist deshalb möglich, weil im Cyberspace des Internets selbst solche Einträge beziehungsweise Informationen, die in den traditionellen Kommunikationsmedien keinen Platz finden, nicht verschwinden. Allein durch die Tatsache, dass sie im Internet vorhanden sind, besitzen sie das grundsätzliche Potential, auch wenn sie lange nicht beachtet werden, irgendwann ein größeres Echo zu finden. Dies kann man mit dem Konzept der „long tail“ von Chris Anderson erklären, der es ursprünglich verwendete, um ein besonderes Phänomen im e-business zu erklären: Es wären demnach nämlich nicht die großen Verkaufsschlager, die „hits“, sondern vielmehr die vielen wenig verkauften Artikel, die „misses“, die den Hauptanteil des Umsatzes von Online-Unternehmen wie Amazon ausmachen würden. ${ }^{18}$ Die Ladenhüter müsste man nach der traditionellen Geschäftslogik aus dem Programm nehmen, um Lagerkosten zu sparen. Aber im e-business sei dies nicht erforderlich, da solche Lagerkosten nicht anfielen. So habe man dort eine möglichst breite Palette, ja alle verfügbaren Artikel im Angebot. Es sei immer möglich, dass sich ein Ladenhüter irgendwann in einen Verkaufsschlager mit großen Umsätzen verwandele.

Wann und wie ein „miss“ zum „hit“ aufsteigen kann, ist, wenn überhaupt, nur bedingt steuerbar. Das gilt für das e-business, aber noch mehr für politische Debatten und Mobilisierungen im Internet, da die Knotenbildung fließender und der Prozess der Kommunikation nicht durch die Sache selbst (wie beim Bücherkauf) bestimmt ist. Diese Undefiniertheit ist Voraussetzung und Ergebnis der Spontanität, mit der sich die Bürger beziehungsweise die Netizens im Internet bewegen. Dies macht gerade die Besonderheit der online-basierten politischen Aktivitäten aus. Wo und wann es zu spontanen Offline-Aktionen kommt, ist nicht vorhersehbar. Dafür sind die so genannten flashmobs nicht das einzige Beispiel. Wie die Erfahrung in Südkorea zeigt, kann selbst eine Website, auf der Kochrezepte ausgetauscht werden, plötzlich in den Mittelpunkt politischer Aktionen rücken.

Solche Phänomene wurden jedoch ungeachtet ihrer Betonung durch Bruce Bimber ${ }^{19}$ in der akademischen Internet-Debatte kaum berücksichtigt. Eine Ursache dafür dürfte darin liegen, dass sich diese Debatte hauptsächlich mit dem Online-Engagement von Politikern, befasst. Aus dieser top-down Perspektive wird die bottom-up Seite der Nutzung des Inter-

18 Im „digital business“ gelte heute deshalb das so genannte Pareto-Prinzip von 80 bis 20 nicht, sondern eben das Gesetz von der „long tail“. In Klammern sei angemerkt, dass Pareto selbst nicht von einem solchen Prinzip gesprochen hat, sondern nur lediglich beobachtet hat, dass Ende des 19. Jahrhunderts in Italien 20 Prozent der Familien etwa 80 Prozent des Vermögens besaßen. Er empfahl deshalb, dass sich die Banken zur Sicherung ihrer Geschäftsgrundlage vornehmlich um diese 20 Prozent kümmern sollten; vgl. Chris Anderson, The Long Tail, www.wired.com/wired/ archive/12.10/tail.html (Abruf am 15. April 2009).

19 Vgl. Bruce Bimber, a.a.O. (Fn. 4). 
nets durch die Bürger kaum beachtet. ${ }^{20}$ Selbst dann, wenn deren Engagement untersucht wird $^{21}$, richtet sich der Blick der Forscher auf konstante, sich wiederholende und wohlorganisierte Aktivitäten der Netizens und übersieht dadurch ihre Spontanität, Unberechenbarkeit und Nichtsteuerbarkeit.

Durch die online-basierten spontanen Aktivitäten bilden sich neue Formen politischer Netzwerke und des Informations- und Meinungsaustauschs. ${ }^{22}$ Die Bürger können auf diese Weise zu einer neuen politischen Kraft werden. Im Online-Kommunikationssystem konstituiert der Prozess der Produktion, Verbreitung, Archivierung und Reproduktion der Informationen eine Beziehung zwischen unzähligen Produzenten und Konsumenten, wobei jeder Teilnehmer sowohl Produzent als auch Konsument sein kann. Jeder kann theoretisch mit jedem und allen in Verbindung treten. Die Hierarchien von Produzenten und Konsumenten, von Informationen und Wertungen werden in einer neuen N:N-Beziehung der Kommunikation aufgehoben. ${ }^{23}$ Die Verbindungen in diesem System beruhen auf der Reziprozität aller Teilnehmer in diesem N:N-Geflecht, dessen Verbindungen sich mit der Zahl der Teilnehmer, also links, exponentiell erhöhen.

Die Dynamik entsteht, weil die Häufigkeit der Verbindungen zwischen verschiedenen Teilnehmern nicht gleichmäßig auf alle verteilt ist, sondern bestimmte Themen, die an bestimmten Punkten („nodes“) zusammenlaufen, häufiger angeklickt/aufgerufen werden, um Informationen einzubringen oder aufzunehmen. Hier gilt das Prinzip der power law distribution. Im Ergebnis kristallisieren sich einige nodes als Knotenpunkte (connector beziehungsweise hub) heraus. So wird der Informationsfluss stark beschleunigt, was zur gemeinsamen Meinungsbildung einer sehr großen Zahl von Teilnehmern führen kann. Es entstehen spontane Themengruppen, die auch offline agieren können. ${ }^{24}$ Spätestens dann

20 Vgl. Rachel Gibson / Wainer Lusoli / Stephen Ward, The Australian Public and Political On-Line: Reinforcing or Reinventing Representation?, in: Australian Journal of Political Science, 43. Jg. (2008), H. 1, S. 111 - 131. Die meisten Studien konzentrieren sich auf Fragen der Nutzung des Internets im Kontext von Wahlen und der Organisierung politischer Kampagnen; vgl. Bruce Bimer / Richard Davis, Campaigning Online: The Internet in U.S. Elections, New York 2003; Paul Herrnson, Congressional Elections: Campaigning at Home and in Washington, Washington D.C. 2004 .

21 Vgl. Paul Ferber / Franz Foltz / Rudy Pugliese, Community Networks and Public Participation: A Forum for Civic Engagement or a Platform for Ranting Irate Malcontents?, in: Bulletin of Science, Technology \& Society, 26. Jg. (2006), H. 5, S. 388 - 397.

22 Manuel Castells schreibt dazu: „As a historical trend, dominant functions and processes in the information age are increasingly organized around networks. Networks constitute the new social morphology of our society, and the diffusion of networking logic substantially modifies the operation and outcomes in process to production, experience, power, and culture. While the networking form of social organization has existed in other times and spaces, the new information technology paradigm provides the material basis for its pervasive expansion throughout the entire social structure." Manuel Carstells, The Information Age: Economy, Society and Culture, Bd. 1: The Rise of the Network Society, London 1996, S. 469.

23 Das Informationsverbreitungssystem von Print- und Rundfunkmedien beruht auf dem Verhältnis von Produzenten und Konsumenten von Informationen, das heißt es beruht auf dem Prinzip der einseitigen Informationsvermittlung. Für die Reziprozität zwischen dem Produzenten und Konsumenten ist hier so gut wie kein Platz, noch weniger für die Reziprozität zwischen Konsumenten.

24 Nach Barabasi und Albert ähnelt dieser Prozess der Selbstorganisierung der online-community einem Naturphänomen, bei dem sich Fraktale in einem chaotischen Prozess in neuer Komplexität formieren, das heißt sich selbst organisieren. Vgl. Albert-Laszlo Barabasi / Reka Albert, a.a.O. (Fn. 17). 
werden sie für Politik und traditionelle Medienöffentlichkeit sichtbar und können diese zwingen, sich mit ihren Auffassungen auseinanderzusetzen. Gegebenenfalls mobilisieren sie auch weitere Bürger online wie offline. Die neuen Medien machen es sogar möglich, dass Netizens online an Offline-Demonstrationen teilnehmen. Die Online- und die mit ihr verbundene Offline-Öffentlichkeit können auf diese Weise den herkömmlichen Begriff der Öffentlichkeit grundlegend verändern: Die Geheimhaltung von Informationen und die Manipulation von „Informationskonsumbürgern“ durch Politik und Medien wird erschwert - während im spontan-chaotischen Mobilisierungsprozess über die Netizens zugleich neue populistische Bewegungen denkbar geworden sind.

Bei der Konstituierung solcher Netzwerke spielt die soziale beziehungsweise wirtschaftliche Position der Produzenten von Informationen interessanterweise keine besonders wichtige Rolle. Wie die Erfahrung in Korea zeigt, hängt die Frage, ob bestimmte Meinungen und Beiträge, die sich online verbreitet haben, bei den Netizens auf breite Zustimmung stoßen oder nicht, in erster Linie von der Qualität der Information ab. Die „Equipotentialität" gehört zu den besonderen Eigenschaften der Online-Öffentlichkeit. Sie besteht darin, dass „every participant has the potential to make a worthwhile contribution, and that a principle of openness therefore offers the best likelihood that such contributions will be made and identified by the wider community "25. Dabei ist die Betonung von Equipotentialität keineswegs als idealistischer Glaube an die grundsätzliche Gleichheit und Gleichwertigkeit aller Ansichten und Beiträge in der politischen Online-Debatte zu missverstehen. Equipotentialität respektiert vielmehr ,the differences between people [...] indeed utilises such differences by harnessing them in its pursuit of broad-based, multiperspectival political deliberation" ${ }^{26}$.

Die hier skizzierten besonderen Eigenschaften der Online-Aktivitäten von Bürgern lassen sich anhand der Erfahrungen Südkoreas während der letzten zehn Jahre empirisch darstellen. In diesem Sinne stellt die dortige elektronische Demokratie ein Testfeld für die theoretischen Überlegungen dar.

\section{Elektronische Demokratie in Korea - ein Testfall}

Seit Mitte der 1990er Jahre hat sich in Südkorea die neue Kommunikationstechnologie rasch verbreitet und das Land eine schnelle Entwicklung der e-democracy erlebt. Die Entstehung einer Online-Öffentlichkeit hat den sozialen Bewegungen eine neue Dimension an Wirkungsmöglichkeiten eröffnet. Erste klare Anzeichen dafür waren bereits 1996 und 1997 in einer der ersten großen E-Aktionen zur Unterstützung des Generalstreiks zu erkennen. Damals ging es um die Reform des Arbeitsgesetzes und die Annullierung des Gesetzes, das die KCIA (Korean Central Information Agency) absicherte. ${ }^{27}$ Über PC-Netzwerke wurden Informationen verbreitet, während sich Ansätze einer alternativen Öffentlichkeit bildeten. ${ }^{28}$

25 Axel Bruns, Life Beyond the Public Sphere: Towards a Networked Model for Political Deliberation, in: Information Polity, 13. Jg. (2008), H. 1-2, S. 65 - 79. S. 71.

26 Ebenda.

27 Vgl. http://strike.nodong.net/strike9697 (Abruf am 25. Juli 2010).

$28 \mathrm{Vgl}$. Byoung-Il Oh, 15 Years of the Information and Communication Movement with the CoExistence of Restrictions and Potentialities, in: Hyeung-Mo Lee (Hrsg.), Korean Civil Society and NGOs 1987-2002, Seoul 2004, S. 471 - 484, S. 437. 
Das Besondere an diesen Aktionen war, dass sie nicht von der Gewerkschaftsführung, sondern von einzelnen, mit der demokratischen Arbeiterbewegung sympathisierenden Nutzern dieser digitalen Netzwerke, die in Korea später Netizens genannt werden sollten, organisiert worden waren. Auch eine der ersten großen Cyberprotestbewegungen, „Anti-NIX“ 1999 und 2000, wurde durch die Initiative einzelner Netizens auf den Weg gebracht. ${ }^{29}$ Die Firma NIX hatte 1999 einen Preis für einen attraktiven Namen für ihre Domain ausgesetzt und erhielt daraufhin 300.000 Vorschläge. Ausgewählt wurde der Vorschlag einer der Firma nahe stehenden Person, die dann auch das Preisgeld von 300 Millionen Won (circa 250.000 Euro) erhielt. Die Netizen hielten das Ganze für Betrug und forderten die Firma auf, sich öffentlich zu entschuldigen und das Preisgeld einer gemeinnützigen Wohltätigkeitsorganisation zu übergeben. Letztlich musste NIX diesen Forderungen nachgeben. Dies war der Beginn einer neuen Art von sozialer Bewegung: Nicht Organisationen, sondern einzelne Netizens - in diesem Fall sogar nur eine einzige Person - mobilisieren Hunderttausende anderer Netizens und werden so zu einer durchsetzungsfähigen sozialen Kraft.

Da die online-basierte Kommunikation für die Wahlen von 2000, 2002 und 2004 eine ausschlaggebende Rolle gespielt hatte, weckte die Entwicklung der e-democracy in Korea in zunehmendem Maße die Aufmerksamkeit der Forschung. Bei der Parlamentswahl 2000, deren Ausgang durch die online-basierte Widerstandsbewegung („rejection-movement“30) vieler NGOs in unerwartet starker Weise beeinflusst wurde, war die enorme Wirkungskraft des Internets als alternatives Medium zur raschen Informationsverbreitung deutlich geworden. Die Erfahrungen von 2002 und 2004 wurden zur Ouvertüre für die Entfaltung neuer Formen der politischen Partizipation von Netizens, die sich nicht mehr nur online, sondern auch offline engagierten. Diese Aktivitäten fanden allerdings keinen Niederschlag in der Wahlbeteilung, dem traditionellen Maßstab für politische Partizipation, die seit der Demokratisierung in Korea 1987 kontinuierlich sinkt (vgl. Abbildung 1).

Diese neuen Formen der politischen Selbstmobilisierung und Partizipation kamen paradigmatisch bei Nosamo, einem Verein zur Unterstützung des damaligen Präsidentschaftskandidaten Roh Moo Hyun, zur Geltung. Deshalb gilt die Präsidentschaftswahl 2002 als Zäsur in der politischen Entwicklung Südkoreas. Nosamo war der erste, spontan entstandene Fanclub für einen Politiker. Dieser organisierte im Cyberspace und auf Wahlveranstaltungen Aktionen für Roh Moo-Hyun. Dabei war Nosamo weder eine Wahlkampforganisation noch ein politisches Organ der Partei Roh Moo-Hyuns, sondern bloß ein Fanclub, der

29 Vgl. http://ihateifree.com (Abruf am 25. Juli 2010).

30 Im Hinblick auf die Parlamentswahl 2000 schlossen sich mehrere hundert NGOs zu einer gemeinsamen Dachorganisation „Chongseon simin yeondae“ (Bürgersolidarität für die Parlamentswahl) zusammen und setzten die persönliche Eignung der Kandidaten für den erstrebten Sitz im Parlament auf ihre politische Agenda. Sie verbanden damit eine „rejection-Bewegung“ gegen die ihren Recherchen zufolge, ungeeigneten und unqualifizierten Kandidaten. Sie forderte die Bürger darüber hinaus zur aktiven politischen Partizipation auf. Neben Unterschriftenaktionen für die Unterstützung ihrer „rejection-Bewegung“ wurden über „Mitizen“ (Mobile Citizen) gemeinsame Aktionen organisiert. Dafür wurden Bürgerorganisationen im Internet verlinkt. Ihre Bulletin Boards System (BBS) ermöglichten auf diese Weise ein breites Diskussionsforum. Ihre vor allem auf Internet basierten Aktivitäten waren so erfolgreich, dass bei der Wahl am 13. April 200059 von 86 Kandidaten auf dieser Liste durchfielen. Mehr dazu siehe Eun-Jeung Lee, Internet und Entstehung einer Gegenöffentlichkeit. Politische Mobilisierung in Korea 2000 - 2004, in: ZParl, 35. Jg. (2005), H. 4, S. $808-823$. 


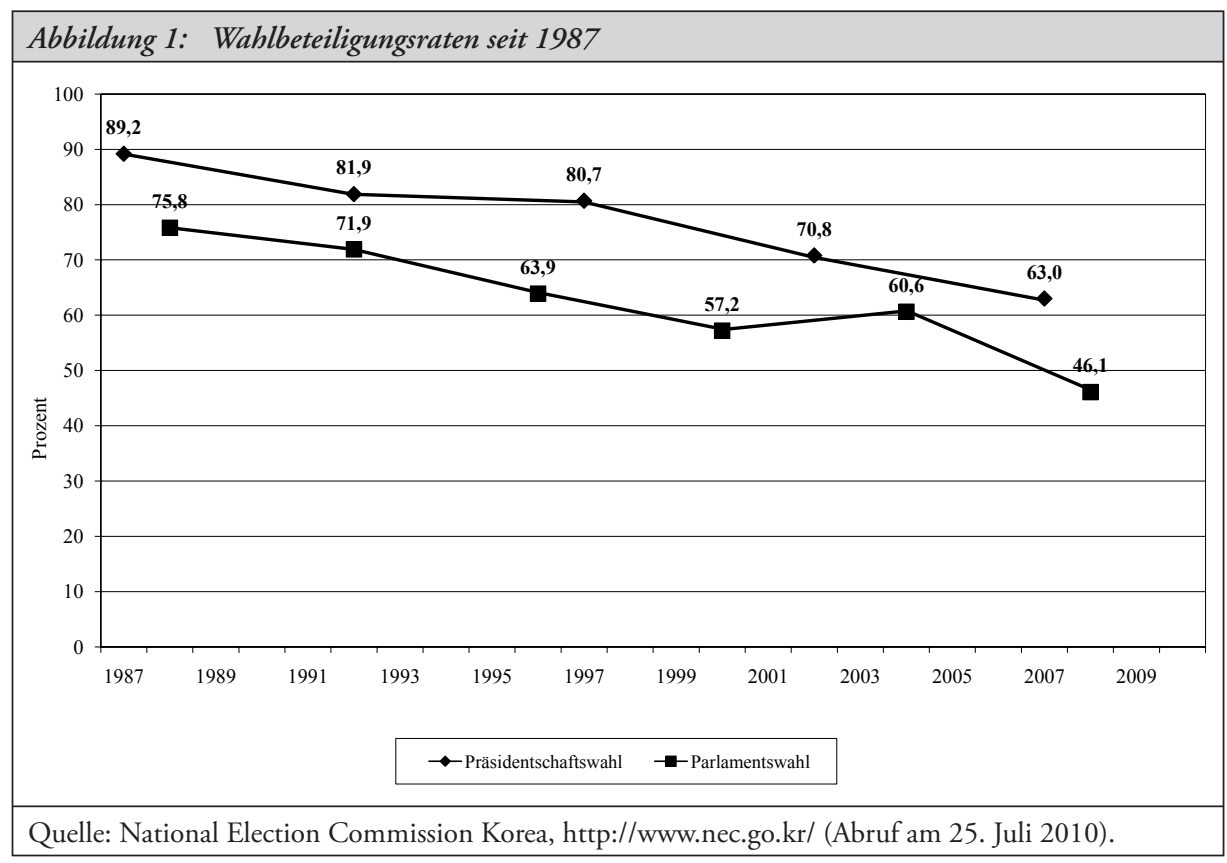

sich ohne Mitwirkung des Kandidaten und der Partei selbst gebildet hatte. Im Juni 2000, nachdem Roh Moo-Hyun wegen des starken Regionalismus in Korea die Parlamentswahl im April verloren hatte, trafen sich etwa hundert Netizens, die online über diesen Fall diskutiert hatten, in einem Internetcafé und gründeten eine politische „e-community“ zur Unterstützung seiner Politik.

Der Fall Nosamo machte deutlich, dass Bürger mehr sein können als nur passive Wähler, die lediglich von Kandidaten und Parteien gelieferte Informationen konsumieren; sie können sich nämlich in aktive Produzenten von Informationen verwandeln. Man könnte hierbei von produktiven Konsumenten des digitalen Kommunikationszeitalters sprechen.

Die wissenschaftliche Diskussion dieser Ereignisse konzentrierte sich auf die Beziehung von Wahlergebnissen und Internet, schenkte aber diesen neuen Formen der politischen Partizipation kaum Beachtung. Diese beschränkte Sichtweise ließ sich neuerlich 2004 in Bezug auf die Online-Protestbewegung gegen die Amtsenthebung von Präsident Roh MooHyun durch das Parlament am 12. März 2004 und auf die kurz darauf folgende Parlamentswahl beobachten. ${ }^{31}$ Insofern kann es nicht überraschen, dass sich das Interesse von Medien und Wissenschaft auch bei der Präsidentschaftswahl im Dezember 2007 und der Parlamentswahl im April 2009 vornehmlich auf die Rolle des Internets richtete, obgleich das Internet bei diesen Wahlen kaum eine Rolle spielte. Viel wichtiger war der Einfluss der drei großen konservativen Zeitungen, die wegen der neuen Internet-Öffentlichkeit angeblich ihre Bedeutung als "gate keeper" verloren hatten. Es ist nicht zu bestreiten, dass sie zu dem überzeugenden Sieg ihres Präsidentschaftskandidaten Lee Myung-Bak und seiner Partei

31 Vgl. ebenda. 
bei der Parlamentswahl beigetragen haben. ${ }^{32}$ Die erfrischende Wirkung des Internets auf die Politik schien damit fürs erste wieder verloren gegangen zu sein.

Dass das nicht wirklich der Fall war, zeigte sich unmittelbar nach der Parlamentswahl in den Kerzendemonstrationen, die das Land mehrere Monate bewegte. Als bekannt wurde, dass Rindfleischimporte aus den USA praktisch ohne Auflagen und Kontrollen wieder zugelassen werden sollten, verbreitete sich im Internet schnell Kritik, die zu massiven Onlineund Offline-Mobilisierungen von Netizens und Bürgern führte. Die Straßen der großen Städte in Südkorea füllten sich von Mai bis Juli zeitweise täglich mit Zigtausenden, an manchen Tagen Hunderttausenden protestierender Bürger. Bemerkenswert ist dabei die Tatsache, dass diese über 100 Tage dauernde Protestbewegung von keiner Organisation angeführt und koordiniert wurde. Stattdessen waren die Initiatoren einzelne Netizens, die über BSE-Gefahren und die hygienischen Bedingungen amerikanischer Schlachthöfe diskutierten. Diese Art der politischen Partizipation, die zur massenhaften Selbstmobilisierung der Bürger führte, unterscheidet sich markant von traditionellen Formen bürgerschaftlicher Teilhabe an politischen Entscheidungen. Zu beobachten waren Prozesse der „Ent-Organisierung" politischer Partizipation im Sinne der Transformation von Interessengruppen in Themengruppen. ${ }^{33}$

Dies war im Zusammenhang mit der Bewegung gegen das Amtsenthebungsverfahren von Präsident Roh Moo-Hyun zum ersten Mal deutlich geworden. Ein Netizen rief wenige Minuten, nachdem die Entscheidung gefallen war, die Bürger zum Protest auf; spontan kamen 15.000 Bürger vor dem Parlament zusammen. Bürgerorganisationen wie Peoplespower 21 klinkten sich ein und koordinierten die weiteren Protestaktionen, die nach acht Tagen mit circa 500.000 Teilnehmern ihren Höhepunkt erreichten. ${ }^{34}$ Demgegenüber waren bei den Kerzendemonstrationen 2008 die Netizens nicht nur die Initiatoren, sondern blieben über mehr als 100 Tage die treibende Kraft der Proteste. Etablierte Bürgerorganisationen konnten ihre Mitglieder zwar zur Teilnahme an den Kerzendemonstrationen aufrufen, durften aber keine Führungsrolle übernehmen. Stattdessen kam es zu einer Selbstmobilisierung der Bürger über circa 1.700 e-communities, die sich in einem um das Problem des Rindfleischimports gescharten, lockeren Netzwerk verbanden. Die Kerzendemonstrationen waren somit der äußere Ausdruck von online-basierten politischen Aktivitäten und zeigten zudem, welche enormen Mobilisierungspotentiale in solchen Netzwerken stecken. ${ }^{35}$

Die Schüler reagierten zuerst auf die Entscheidung, US-Rindfleischimporte wieder zuzulassen. Sie hatten bereits online und per SMS eine heftige Debatte über die am 15. April

32 Vgl. Werner Kamppeter / Eun-Jeung Lee, Demokratie im Internet-Zeitalter. Vier Monate Kerzendemonstrationen in Korea. Hintergründe und Ursachen, in: Friedrich-Ebert-Stiftung Berichte, http://library.fes.de/pdf-files/iez/05467-b.pdf (Abruf am 28. Juni 2009).

33 Vgl. Bruce Bimber, a.a.O. (Fn. 4).

34 Vgl. Eun-Jeung Lee, Internetpower und soziale Bewegung in Korea, in: Welttrend, 13. Jg. (2005), H. 48, S. $53-65$.

35 Die folgende Darstellung über die Kerzendemonstration von 2008 beruht auf die teilnehmende Beobachtung und Dokumentationen wie P'yein Agora, Taehanminguk sangsik sajŏn agora [Enziklopädie Korea common sense agora], Seoul 2008; Chi-hŭi Kwŏn u.a., Ch’otpuli minjujuŭida [Kerzen sind Demokratie], Seoul 2008; P’yŏnjippu (Hrsg.), Agoraesŏ sŏulgwangjangkkaji kkŏjiji annŭn ch'otpul [Von agora zum Seoul Plaza nicht zu löschende Kerzen], Seoul 2008; Gu-hyŏn Nam u.a., Taehan'mingug-ŭn minjugonghwagugida [Korea ist eine demokratische Republik], Seoul 2008. 
bekannt gemachte so genannte „liberale“ Erziehungspolitik der neuen Regierung geführt und kritisiert, dass diese den Druck auf die Schüler erhöhen und Kinder aus Unter- und Mittelschichten weiter benachteiligen würde. Als am 23. April die Entscheidung über Fleischimporte bekannt wurde, verbreiteten sich über die Website „mich'inso dat net“ (www. michincow.net) rasch Informationen über BSE. Als Präsident Lee Myung-Bak die Öffentlichkeit und seine Kritiker mit dem Hinweis zu belehren versuchte, dass er seine Bürger lediglich mit gutem und preiswertem Rindfleisch versorgen wolle und wem dies nicht gefalle, der müsse es ja nicht essen, entflammte er damit die Wut der Schüler, insbesondere auch, weil sie meinten, sie selbst würden qua Schulspeisung von der Regierung zu den Hauptkonsumenten dieses „BSE-Rindfleisches“ gemacht. Entsprechend stieß die OnlinePetition eines Schülers, die dieser am 6. April 2009 auf der Portalseite Daum unter der ID „andante“ vorlegte, auf schnelle und breite Unterstützung. ${ }^{36}$ Obwohl sich diese Petition nicht auf der Hauptseite von Daum befand, wurde sie innerhalb weniger Tage von mehr als 100.000 Netizens unterschrieben. Am 28. April wurde auf der Debattenseite von Daum Agora geäußert ${ }^{37}$, dass man mit Online-Petitionen nicht viel bewirken könne, aber Kerzendemonstrationen ein effektiveres Protest-Mittel wären. Zu diesem Zeitpunkt konnte niemand ahnen, welche Resonanz dieser Vorschlag finden würde und welches Ausmaß an Offline-Aktivitäten er bewirken würde. Diese Debatten im Internet wurden von der Regierung und den Massenmedien kaum beachtet.

An den ersten Kerzendemonstrationen im Zentrum von Seoul am 2. und 3. Mai nahmen einige Tausend Schüler teil. An den folgenden Tagen gesellten sich ihnen auch andere Bürger hinzu, doch blieb die Präsenz der Schüler in ihren Uniformen unübersehbar. Es wurde schnell online wie offline ein Konsens gefunden, die Kerzendemonstrationen so lange fortzusetzen, bis die Regierung mit den USA über die Rindfleischimporte neu verhandeln und die Entscheidung vom April rückgängig machen würde. Darüber hinaus hatten bis zum 6. Mai 500.000 und bis zum 13. Mai eine Million Netizens eine Online-Petition unterschrieben, in der sie die Amtsenthebung des Präsidenten forderten.

Die Regierung bezeichnete die sich online schnell verbreitende Kritik an ihrer Entscheidung, US-Rindfleischimporte wieder zuzulassen, als „Internet-Horrorgeschichte“ und kündigte an, den Schüler, der die Online-Petition für eine Amtsenthebung des Präsidenten vorgeschlagen hatte, zu verhaften. Daraufhin kam es massenhaft zu Selbstanzeigen von Netizens auf der Homepage der Polizei: Sie seien Andante und die Polizei müsse sie bitteschön verhaften. ${ }^{38}$ Plattform für diese Aktion bildete die Diskussionsseite von Daum Agora. ${ }^{39}$

Von all dem nahmen die konservativen Tageszeitungen und die größte Internet-Portalseite Südkoreas, „Naver“, kaum Notiz. ${ }^{40}$ Dagegen betonten sie die Ungesetzlichkeit der Kerzendemonstrationen. Dass die Wahrnehmung und Darstellung von politischen Ereignissen in konservativen Tageszeitungen und alternativen Internetmedien ganz unterschiedlich ausfallen, ist in Korea nichts Neues. Dies war auch 2002 und 2004 zu beobachten gewesen. ${ }^{41}$ Dennoch ist das Internet nicht an sich ein alternatives Medium. Wie Bimber

36 Vgl. http://agora.media.daum.net/petition/ (Abruf am 25. Juli 2010).

37 Vgl. http://agora.media.daum.net/debate/ (Abruf am 25. Juli 2010).

38 Vgl. P'yein Agora, a.a.O. (Fn. 35).

39 Vgl. http://agora.media.daum.net/debate/ (Abruf am 25. Juli 2010).

40 Vgl. http://www.naver.com/ (Abruf am 25. Juli 2010).

41 Vgl. Eun-Jeung Lee, a.a.O. (Fn. 30). 
betont, sind die Inhalte wichtiger als das technische Medium. ${ }^{42}$ Das wurde 2008 deutlich erkennbar. Die Netizens zogen nämlich im Cyberspace weiter, um die „richtige Plattform“ für ihre Debatten zu finden: Naver war über Jahre das führende Internetportal in Korea gewesen, im Juli 2007 liefen über 78 Prozent der Internetabfragen darüber. Im Zusammenhang mit den oben beschriebenen Online- und Offline-Ereignissen entstand unter den Netizens der Verdacht, dass Naver Informationen manipuliere. In der Folge wurde vorgeschlagen, das Portal nicht mehr als Startseite zu benutzen, so dass viele koreanische „digitale Nomaden" sich eine andere „Oase" suchten. Darüber hinaus bot Naver keine gut funktionierende und offene Plattform wie Daum Agora.

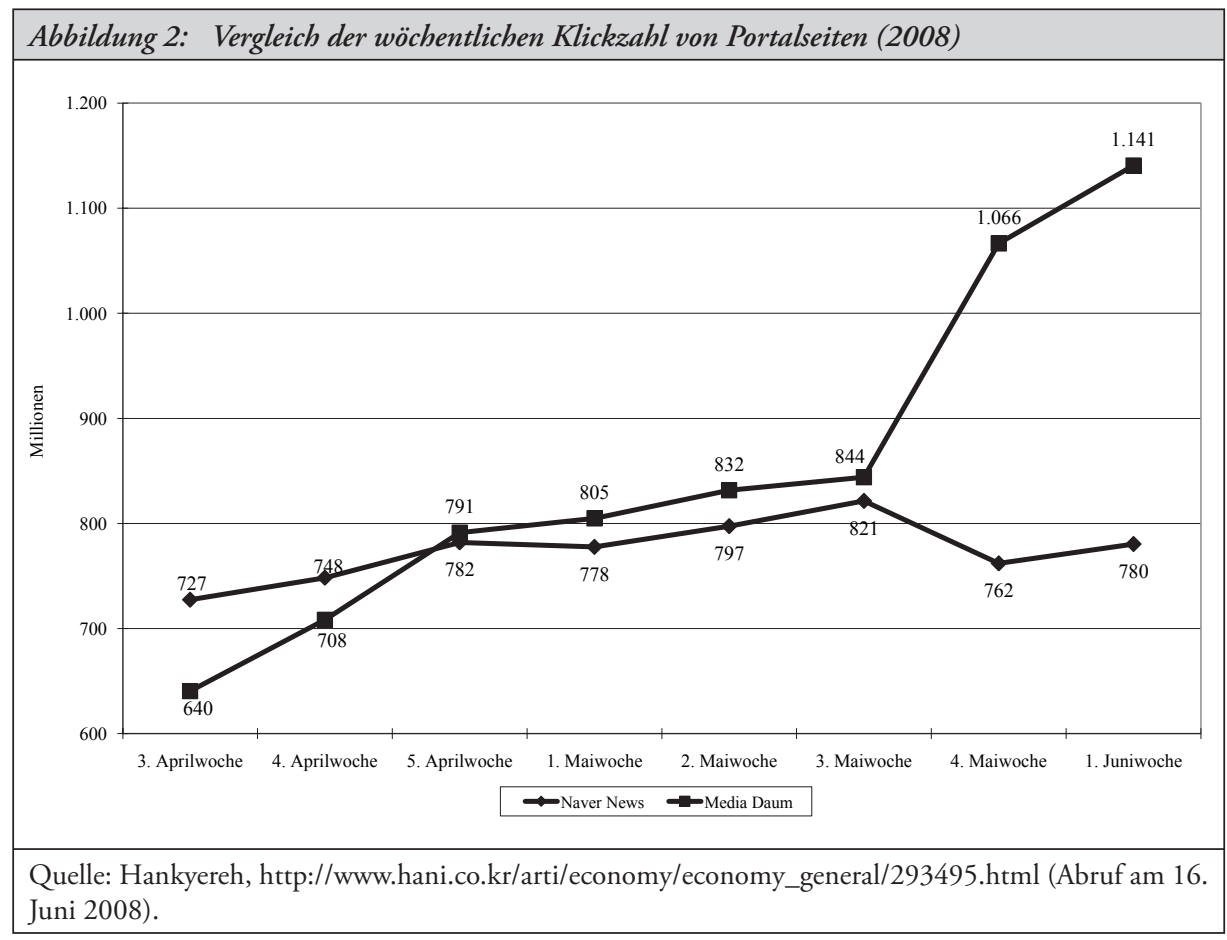

Abbildung 2 zeigt, dass sich Daum ab der dritten Maiwoche, als sich die Kerzendemonstrationen auf immer breitere Bevölkerungsschichten ausweiteten, schnell wachsender Beliebtheit erfreute, während die Zahl der Naver-Nutzer leicht zurückging. Das deutet darauf hin, dass der „Exodus“ nicht wirklich stattfand. Man darf vermuten, dass die Netizens weiterhin auf Naver zurückgriffen und sich bei Daum einklickten, um sich über die laufenden Debatten und die Demonstrationen zu informieren beziehungsweise an ihnen teilzunehmen. Viele Netizens sagen von sich selbst, dass sie zum ersten Mal im Zusammenhang mit diesen Protesten gegen die Rindfleischimporte ein aktives politisches Interesse entwickelten. Viele Schüler, junge Frauen und junge Mütter waren bis dahin nicht mit Politik in Berührung gekommen, insbesondere nicht mit jenen politischen Netzwerken von Studenten, Intellek-

42 Vgl. Bruce Bimber, a.a.O. (Fn. 4). 
tuellen und Bürgeraktivisten, die in Südkorea jahrzehntelang die Demokratiebewegung getragen haben.

Auf besonders große Resonanz stießen Vorschläge für die Gestaltung der Protestaktionen zum Beispiel aus einem Online-Café von Hausfrauen für den Austausch von Kochrezepten $^{43}$, aus einer Online-Gruppe junger modeinteressierter Frauen ${ }^{44}$ oder aus einer Informationsseite für die Ausstattung von Wohnungen. ${ }^{45}$ Die Diskussionsseite von Daum Agora wirkte wie eine Werkstatt interessanter Ideen für die Online- und Offline-Aktionen. Zugleich bildete sie eine offene Plattform für alle, die sich für das Thema Rindfleischimporte interessierten, und verkörperte als solche eine neue Form eines politischen Netzwerkes. Sie wurde schließlich zum wichtigsten Knotenpunkt (connector) der Kerzendemonstrationen.

Die Seite Agora von Daum besteht aus vier Rubriken: Diskussion („t'oronbang“), Erzählen (,iyagi“), Fotos („,chŭlbodŭ“) und Petitionen („ch’ŏngwŏn“). Das Besondere von Agora sind die Diskussions- und die Petitionsseite. Auf der Petitionsseite hatte der Schüler mit der ID Andante die Forderung, Präsident Lee Myung-Bak seines Amtes zu entheben, eingebracht. Auf der Diskussionsseite befinden sich dreizehn „Bulletin Boards (BBS)“ zu unterschiedlichen Themen. Beiträge, die von Besuchern dieser Seite durch einen Klick am häufigsten weiterempfohlen werden, kommen auf die Hauptseite von Agora, der prominenteste sogar auf die Hauptseite von Daum. Zwischen Mai und Juli 2008 behandelten über 90 Prozent der Diskussionsbeiträge von Agora das Thema der US-Rindfleischimporte, während es einer über die Kerzendemonstrationen stets als „bester Beitrag“ auf die Hauptseite schaffte.

Ihre Autoren waren so gut wie nie Experten. Wäre es eine Debatte von Fachleuten gewesen, hätte sie kaum ein so großes Echo gefunden. Da die Beiträge von ganz normalen Bürgern stammten und dennoch als „beste Meinung“ auf den Hauptseiten zu finden waren, überdies zigtausend Male angeklickt wurden, fassten viele den Mut, sich auch selbst an dieser Diskussion zu beteiligen. Dass ihre Meinungen nicht gleich gelöscht, sondern von anderen gelesen und kommentiert wurden, war für viele, die es gewohnt waren, nur einseitig Konsumenten von Medieninformationen zu sein, eine ganz neue Erfahrung.

Es gibt in Korea und anderswo genügend Forscher, Politiker und Intellektuelle, die den Online-Diskussionen der Netizens und „Massenintelligenz“ überhaupt mit Skepsis begegnen. Auch Daum Agora sieht sich häufig scharfer Kritik und dem Populismusvorwurf ausgesetzt. Die Idee der Equipotentialität ist solchen Kritikern fremd. Sie sehen darin lediglich eine Verdummung der Masse und „anti-intellektuelle Hetze“. Hinter der skeptischen Haltung stehen nicht selten geistige Arroganz und das Überlegenheitsgefühl der Kritiker. Ein charakteristisches Beispiel dafür ist die Behauptung Adam Soboczynskis, der in seinem Artikel „Netz als Feind“ schreibt, dass „der Intellektuelle aus der Mehrheitsdemokratie geistesaristokratisch herausragt “46. Er ignoriert dabei aber die Tatsache, dass es sogar empirisch

43 Vgl. http://www.82cook.com (Abruf am 25. Juli 2010).

44 Vgl. http://cafe.daum.net/SoulDresser (Abruf am 25. Juli 2010).

45 Vgl. http://www.lemonterrace.com/ (Abruf am 25. Juli 2010).

46 Adam Soboczynski, Das Netz als Feind. Warum der Intellektuelle im Internet mit Hass verfolgt wird, in: Die Zeit vom 20. Mai 2009, http://www.zeit.de/2009/22/Der-Intellektuelle (Abruf am 17. Februar 2010). 
nachgewiesen ist, dass die Masse nicht unbedingt weniger intelligent ist als die Experten. ${ }^{47}$ Anders als die Skeptiker betrachten Hardt und Negri mit großer Sympathie das Phänomen Massenintelligenz; sie bezeichnen dies unter Rückgriff auf Karl Marx als ,general intellect“. Es seien eben „die wissenschaftlichen, affektiven und sprachlichen Kräfte der Menge“, die die gesellschaftlichen Produktionsbedingungen in aggressiver Weise veränderten. „Das Feld, auf dem sich die Menge die Produktivkräfte wieder aneignet, ist ein Feld radikaler Metamorphosen - Schauplatz eines schöpferischen Vorgangs. “48

Auch die Kerzendemonstrationen in Korea könnten als ein solches Feld interpretiert werden. Hardt / Negri sehen die Massenintelligenz zu verantwortlichem und vernünftigem Handeln in der Lage. Das konnte man anhand der Reaktionen der Netizens und Demonstranten gegenüber der Regierung beobachten. Als diese sich während der Demonstrationen, die im Mai und Juni bis zu einer Million Menschen auf die Straßen brachten, hinter Containermauern einigelte, wurde in Daum Agora heftig über die Frage debattiert, ob man diese Mauer überwinden und bis zum Präsidentenpalast marschieren sollte oder nicht. Im Ergebnis wollte man diese Mauer aber nicht physisch überwinden. Ungeachtet der „nackten Gewalt“ seitens der Regierung sollten sich die Demonstranten als „reife Bürger“ verhalten. Die Meinungsäußerung „Wir brauchen die Kraft der Phantasie, nicht die physische Gewalt" wurde innerhalb weniger Stunden über 100.000 Mal angeklickt ${ }^{49}$, denn auch Mütter mit Kinderwagen sollten an den Demonstrationen teilnehmen können. In der Tat trugen die Kerzendemonstrationen überwiegend Volksfestcharakter.

Während der drei Monate von Mai bis Juli 2008 konnte jeder, der sich darüber informieren wollte, dies über Websites wie Daum Agora, OhmyNews (www.ohmynews.com), Hankyoreh (www.hani.co.kr), 82cook.com (www.82cook.com) und lemonterrace (www. lemonterrace.com) tun. Diejenigen, die gern an den Kerzendemonstrationen teilgenommen hätten, dies aber nicht konnten, hatten daran dank Web 2.0 indirekt die Möglichkeit, denn es waren meist sehr viele Camcorder- und Handy-Reporter unterwegs, die ihre Aufnahmen ins Internet übertrugen. Die so ausgerüsteten Netizens wurden zu Produzenten

47 In Deutschland und England wird dies anhand des Vergleichs zwischen Wikipedia und Encyclopædia Britannica beziehungsweise Brockhaus demonstrativ zur Schau gestellt. Im Dezember 2005 ließ die wissenschaftliche Zeitschrift „Natur" die Einträge in Wikipedia und Encyclopædia Britannica im naturwissenschaftlichen Bereich vergleichen. Danach lag die Encyclopædia Britannica im Hinblick auf Korrektheit und Editionsqualität zwar knapp vorn, aber in Wikipedia wurden die festgestellten Fehler sofort korrigiert, während die Nutzer von Encyclopædia Britannica bis zur nächsten neuen Auflage dieses Werkes warten mussten. Die Kritiker gegen Massenintelligenz bemängeln jedoch im Nachhinein, dass Wikipedia seine Stärke im naturwissenschaftlichen Bereich habe, bei sozial- und geisteswissenschaftlichen Themen schwächer und bei politischen sowie historischen Themen gar nicht akzeptabel sei. 2007 machte die Zeitschrift "Stern" in Deutschland den Test, Wikipedia und Brockhaus zu vergleichen. Dafür wurden 50 zufällig ausgewählte Einträge aus den Fachgebieten Politik, Wirtschaft, Sport, Wissenschaft, Kultur, Unterhaltung, Erdkunde, Medizin, Geschichte und Religion nach den Kriterien wie Richtigkeit, Vollständigkeit, Aktualität und Verständlichkeit überprüft und mit Noten bewertet. Dabei erzielte Wikipedia in allen Bereichen eine Durchschnittsnote von 1,7. Die Einträge zu den gleichen Stichworten im Online-Brockhaus erreichten lediglich eine Durchschnittsnote von 2,7; Frank Patalong, Wikipedia schlägt die Profis, Spiegel online, 17. Dezember 2007, http://www.spiegel. de/netzwelt/web/0,1518,521457,00.html (Abruf am 25. Juli 2010).

48 Michael Hardt / Antonio Negri, Empire, Frankfurt am Main 2002, S. 374.

49 P'yein Agora, a.a.O. (Fn. 35), S. 137. 
von Internetfernsehprogrammen. Die wichtigste Website für diese Übertragungen war Afreeca.com. Sie wurde seit Mai 2008 täglich mehrere Millionen Mal aufgerufen. Dort konnte man an manchen Tagen weit mehr als 1.000 Live-Übertragungen der Demonstrationen im ganzen Land aufrufen; dazu, ebenso ohne Gebühren, Archivaufnahmen und -filme.

Daran wird deutlich, wie fließend die Übergänge zwischen Online- und Offline-Aktivitäten waren. Dies zeigte sich etwa auch, als ein Redner der Kerzendemonstration am 10. Juni den Online-Teilnehmern/Beobachtern an diesen Veranstaltungen vorschlug, unmittelbar die Homepage des Präsidialamtes aufzurufen. Dadurch sollte dem Präsidenten demonstriert werden, dass das ganze Land online wie offline gegen seine Politik protestierte. Innerhalb einer Minute brach der Server des Präsidialamtes wegen Überlastung zusammen.

Diese direkt handelnden Netizen-Bürger wurden von Soziologen in Korea als die Verwirklichung der „Multitude“ im Sinne von Hardt und Negri interpretiert: „Die Bürger, die mit Kerzen auf die Straße kamen, waren nicht mehr eine einfache Masse. [...] Sie waren eine aktiv partizipierende Multitude. Wissenschaftler definieren Multitude als kluge Masse. Sie tritt als handelndes Subjekt auf, das seine Werte selbst durchsetzt und konkrete Bedürfnisse hat. Eine Multitude, die mit digitaler Technologie gut vertraut ist, akzeptiert keine Autorität. Die Identität dieser Multitude ist mehrschichtiger als die Identität des Volkes, der Bürger und der Individuen. So gibt es bei den Kerzendemonstrationen keine Führungsstruktur. Kein Befehl wird akzeptiert. Multitude agiert zwar individuell, handelt aber gemeinsam gegenüber einem Thema. Sie ist Einheit und Vielfalt. “50

Die Frage, ob man die Netizen-Bürger Koreas mit dem Begriff „Multitude“ adäquat erfassen kann, bedarf sicherlich einer weitergehenden Analyse, nicht zuletzt deshalb, weil der Begriff selbst noch umstritten ist und seine politischen Wirkungsmöglichkeiten bisher nur selten thematisiert worden sind. Dies kann im Rahmen dieses Beitrags nur kurz skizziert werden.

Hardt und Negri definieren Multitude als „Singularitäten, die gemeinsam handeln“ ${ }^{\text {. }}$. Sie soll eben „kluge Masse“ sein, die in der Lage ist, gemeinsam Entscheidungen zu treffen und danach zu handeln. Als solche ist sie nicht mit dem Begriff der Masse gleich zu stellen, den die Massenpsychologie als „einen wilden, ,irrationalen’ Ausbruch von Gewalt“ beschreibt, "der sich durch eine imitatio affecti selber speist und vorantreibt" ${ }^{2}$. Die neuen Typen der sozialen Bewegungen in den letzten Jahrzehnten haben immer wieder gezeigt, dass die Massenmobilisierung im digitalen Informationszeitalter keineswegs mit einem „wilden, irrationalen Ausbruch von Gewalt“ gleichzusetzen ist. Die technischen Möglichkeiten für einen breiten Informations- und Meinungsaustausch haben sicher eine neue Entwicklungsstufe für soziale Bewegungen ermöglicht. Die Menge bildet mit Hilfe der neuen Kommunikationstechnologie nunmehr eine „klug handelnde Masse“. Insofern vermag Multitude im Unterschied zum Massenbegriff diesen Aspekt darzustellen. Problematischer wird die Anwendung des Begriffs jedoch, wenn Multitude auch als supranationale Organisationsform verstanden wird, denn nur wenige soziale Bewegungen weisen transnationalen

50 Mun-jae Yi, Sejongno hanbokp'an kanghan minjujuŭi pulssirŭl poatta [Mitten in Sejongno sah ich den Keim für starke Demokratie], in: Sisa vom 9. Juni 2009, http://www.sisain.co.kr/news/ articleView.html?idxno=2190 (Abruf am 25. Juli 2010).

51 Michael Hardt / Antonio Negri, a.a.O. (Fn. 48), S. 123.

52 Slavoj Zizek, Körperlose Organe. Bausteine für eine Begegnung zwischen Deleuze und Lacan, Frankfurt am Main 2005, S. 57. 
Charakter auf. Die Kerzendemonstration im Jahre 2008 in Korea war ohne Zweifel eine stark nationalistisch geprägte Widerstandsbewegung, deren typische Merkmale in ihrer Themenorientiertheit, aber auch in der Spontanität und Nicht-Berechenbarkeit lagen. Nicht zuletzt deshalb scheint es nur unter starken Vorbehalten akzeptabel, im Falle der Netizen-Bürger Koreas von Multitude zu reden.

Allerdings ist auch deutlich geworden, dass online-basierte Bürgeraktionen durchaus über einen längeren Zeitraum hinweg möglich sind. Als Schwäche erweist es sich dabei, dass sie über keine wirksamen Mittel gegen repressive Maßnahmen des Staates verfügen. So können sich die nicht organisierten Netizen-Bürger kaum gegen eine Regierung wehren, die „Drahtzieher der Demonstrationen“ verhaften lässt und die Online-Debatte durch ein neues Mediengesetz, das die Verwendung der richtigen Namen der Netizen und ihrer Personalnummern vorschreibt, besser kontrollieren will. Tatsächlich gelangte im Herbst 2008 wieder aus den USA importiertes Rindfleisch auf den koreanischen Markt, so dass der Schluss nahe liegt, dass die direkten Netizen-Bürger-Aktionen letztendlich doch gescheitert sind. Dennoch ist zu fragen, welche längerfristige Bedeutung diese Erfahrungen für die weitere Entwicklung der Demokratie in Korea haben werden.

Aus politikwissenschaftlicher Perspektive ist der koreanische Fall vor allem deshalb interessant, weil mithilfe der modernen Kommunikationstechnologien neue Formen der politischen Netzwerkbildung entstanden sind, die die Struktur der Öffentlichkeit verändert und die üblichen politischen Akteure zumindest erheblich verunsichert haben. Dabei gab es für die Bildung dieser Netzwerke keine bestimmte führende beziehungsweise treibende Kraft. Stattdessen bildeten sich auf Portalseiten wie Daum Agora Knotenpunkte, an denen eine sehr große Zahl von Netizens in Kontakt kam. Im Umfeld dieser Seite entstanden weit ausgreifende Netzwerke, deren Teilnehmer miteinander diskutierten und sich gelegentlich zu politischen Aktionen offline entschieden.

\section{Wikipedia-Demokratie: Chance für die repräsentative Demokratie?}

An den koreanischen Erfahrungen ist zu erkennen, dass sich der Bürger im Zeitalter des Internets nicht mehr nur auf seine Rolle als Wähler reduzieren lässt. Mit der Entwicklung der Kommunikationstechnologie hat das weitgehende Informationsmonopol der politischen und wirtschaftlichen Eliten an Bedeutung verloren. Es ist nunmehr denkbar geworden, dass im Internet unter breiter Beteiligung der Netizens eine sachliche Diskussion auch komplexer inhaltlicher und politischer Probleme möglich ist. In diesem Sinne könnte man von einer Wikipedia-Demokratie sprechen.

Diese verstärkt letztlich die Tendenz des entbürokratisierten Pluralismus im Zeitalter des Internets. ${ }^{53}$ Das muss nicht bedeuten, dass die Wikipedia-Demokratie die repräsentative Demokratie ersetzen könnte, denn ihr Wesen liegt in der Themen-Orientiertheit. Diese Eigenschaft ist mit dem sich wiederholenden und komplexen Geschäft der täglichen Politik nur schwer vereinbar, kann allerdings in einer repräsentativen Demokratie, die unter Politikverdrossenheit leidet, durchaus als Ergänzung betrachtet werden. Wie viele Industrienationen leidet auch Korea unter einer kontinuierlich sinkenden Wahlbeteiligung und einer

53 Vgl. Bruce Bimber, a.a.O. (Fn. 4). 
immer geringer werdenden Akzeptanz der Parteien, obwohl es sich seine Demokratie vor noch gar nicht langer Zeit blutig erkämpft hat. Unter diesen Umständen kann man die direkten Aktionen von Netizen-Bürgern, die spontan politische Netzwerke bilden und ihre Meinungen online und offline zum Ausdruck bringen, als ein neues Legitimitätspotential durchaus positiv bewerten. Die institutionalisierte Politik der repräsentativen Demokratie erhält auf diese Weise einen neuen „Kontrolleur“ und damit eine neue Dynamik im digitalen Zeitalter. Wie Arthur Rosenberg meint: Die „Demokratie als ein Ding an sich, als eine formale Abstraktion existiert im geschichtlichen Leben nicht, sondern die Demokratie ist immer eine bestimmte politische Bewegung, getragen von bestimmten gesellschaftlichen Kräften und Klassen, die um bestimmte Ziele kämpfen. Ein demokratischer Staat ist demgemäß ein Staat, in dem die demokratische Bewegung die Herrschaft hat "54. Die NetizenBürger stellen eben diese neue „Bewegung“ dar.

Allerdings treffen die direkten Aktionen der Netizen-Bürger bei den traditionellen Akteuren, die sozusagen noch im Rahmen von analogen Kommunikationsmethoden denken, auf Ablehnung, da sie ihnen nur chaotisch, anarchisch, spontan und nichtsteuerbar erscheinen. Die Netizens können wie im koreanischen Fall sogar durch Einschränkungen ihrer Freiheit bekämpft werden. Ob solche Fesseln und Kontrollen technisch überhaupt wirkungsvoll und auf Dauer angelegt sein können, ist eine offene Frage. Jedenfalls kann man argumentieren, dass diese Formen der politischen Netzwerkbildung im Zeitalter der neuen digitalen Kommunikationstechnologie mit ihren besonderen Eigenschaften, also ihrer „Spontanität“ und „Nichtsteuerbarkeit“ nicht als „Gegner der Demokratie“, sondern als Ergänzung und Stärkung der in ihrer öffentlichen Akzeptanz schwächelnden repräsentativen Demokratie gesehen werden sollten.

54 Arthur Rosenberg, Demokratie und Sozialismus, Frankfurt am Main 1988, S. 303. 